\title{
Stability and Convergence of Solutions to Volterra Integral Equations on Time Scales
}

\author{
Eleonora Messina ${ }^{1}$ and Antonia Vecchio ${ }^{2}$ \\ ${ }^{1}$ Dipartimento di Matematica e Applicazioni, Università degli Studi di Napoli "Federico II", Via Cintia, 80126 Napoli, Italy \\ ${ }^{2}$ National Research Council of Italy (CNR), Institute for Computational Application Mauro Picone, Via P. Castellino 111, \\ 80131 Naples, Italy
}

Correspondence should be addressed to Antonia Vecchio; antonia.vecchio@cnr.it

Received 6 April 2015; Accepted 1 June 2015

Academic Editor: Allan C. Peterson

Copyright (C) 2015 E. Messina and A. Vecchio. This is an open access article distributed under the Creative Commons Attribution License, which permits unrestricted use, distribution, and reproduction in any medium, provided the original work is properly cited.

We consider Volterra integral equations on time scales and present our study about the long time behavior of their solutions. We provide sufficient conditions for the stability and investigate the convergence properties when the kernel of the equations vanishes at infinity.

\section{Introduction}

In this paper we consider the Volterra integral equations (VIEs) on time scales of the type

$$
\begin{aligned}
x(t)=f(t)+\int_{t_{0}}^{t} k(t, s) x(s) \Delta s, & \\
& t \in\left[t_{0},+\infty\right)_{\mathbb{T}}=\left[t_{0},+\infty\right) \cap \mathbb{T},
\end{aligned}
$$

where $\mathbb{T}$ is a time scale, which is a nonempty, closed subset of $\mathbb{R}$. In (1) $t_{0} \in \mathbb{T}$, the integral sign has to be intended as a deltaintegral (see Definition 4 in Section 2), and we assume that the given real-valued functions $f(t)$ and $k(t, s)$ are defined in $\left[t_{0},+\infty\right)_{\mathbb{T}}$ and $\left[t_{0},+\infty\right)_{\mathbb{T}} \times\left[t_{0},+\infty\right)_{\mathbb{T}}$, respectively.

In the following section we will give examples of time scales; here we observe that the most popular examples are $\mathbb{T}=\mathbb{R}$ and $\mathbb{T}=\mathbb{Z}$. When $\mathbb{T}=\mathbb{R},(1)$ takes the form

$$
x(t)=f(t)+\int_{t_{0}}^{t} k(t, s) x(s) d s, \quad t \in\left[t_{0},+\infty\right),
$$

of the classical VIE and when $\mathbb{T}=\mathbb{Z}$, we get the explicit Volterra discrete equation

$$
x(t)=f(t)+\sum_{s=t_{0}}^{t-1} k(t, s) x(s), \quad t \in\left[t_{0},+\infty\right)_{\mathbb{Z}} .
$$

So, all the results proved on the general time scale include results for both integral and explicit discrete Volterra equations.

A generalized differential and integral calculus on time scales was developed for the first time by Hilger in [1], where he put the basis for establishing the theory of dynamical equations (delta-derivative equations) over very general time scales. This theory has received great attention [2-4] in order to address many realistic continuous-discrete models in biological and economic applications and to furnish a theoretical framework for developing a unifying analysis. In particular, in [5], a qualitative study of the solutions to nonlinear dynamic equations is described as well as an application to an economic model.

More recently, there has been a growing interest in Volterra integral equations on time scales as they represent a powerful instrument for the mathematical representation of memory dependent phenomena in population dynamic, economy, and so forth. Therefore, this theory has been extended to the integral operator. A pioneering research on this subject is [6], where the main results concerning the existence, uniqueness, and boundedness on compact intervals are presented. After that, a Volterra theory on time scales has been developed and it is still evolving; see, for example, [6-10] and the bibliography therein. 
In [6] a very accurate analysis of the qualitative behavior of the solutions of both linear and nonlinear problems on noncompact intervals is given. In case of linear problems it has been proved that if $f$ is bounded and $\int_{t_{0}}^{t}|k(t, s)| \Delta s<1$, the solution $x(t)$ of (1) is bounded, which means that it is stable with respect to bounded perturbations. In this paper we prove the stability of solutions to VIEs on time scales under more general hypotheses on the delta-integral of the kernel $k$. Hence, we assume that there exists a $\bar{t}<+\infty$ such that $\int_{\bar{t}}^{t}|k(t, s)| \Delta s<1$, for all $t>\bar{t}$, and we study how the freedom before $\bar{t}$ affects the solution over the entire interval $\left[t_{0},+\infty\right]_{\mathbb{T}}$. The investigation carried out here represents an extension of a result already known both for continuous VIEs (see, e.g., [11, Ch. 9] and for discrete implicit Volterra equations (see [12]). However, the technique used in the proof is different and takes inspiration from $[6,13]$.

Moreover, when the kernel $k$ vanishes at infinity, we study the asymptotic behavior of the solution $x(t)$. In the particular case of discrete equation this result has already been proved by analogous techniques by Györi and Reynolds in [12].

The paper is organized as follows. In Section 2 we introduce some basic material needed in the paper. In Section 3 we define the linear model problem and obtain a bound for its solution. Furthermore, in case of vanishing kernel, we prove that the solution of (1) tends to a finite limit if the forcing function $f(t)$ tends to $f_{\infty}<+\infty$ as $t \rightarrow+\infty$. In Section 4 an extension of the previous results to a Hammerstein type nonlinear equation is shown; in Section 5 some examples are given and Section 6 contains our concluding remarks.

\section{Background Material}

In this section we will recall some definitions and theorems that will be useful in the following (see [1-3] and the bibliography therein).

As already mentioned in Section 1, a time scale $\mathbb{T}$ is any closed subset of $\mathbb{R}$.

We assume that the topology in $\mathbb{T}$ is inherited from the standard one in $\mathbb{R}$.

Definition 1. For all $t \in \mathbb{T}$ and $t<\sup \mathbb{T}$, the forward jump operator is

$$
\sigma(t)=\{\inf \tau>t: \tau \in \mathbb{T}\}
$$

and, for $t \in \mathbb{T}$ and $t>\inf \mathbb{T}$, the backward jump operator is

$$
\rho(t)=\{\sup \tau<t: \tau \in \mathbb{T}\}
$$

If $\sigma(t)>t$, the point $t$ is said to be right-scattered $(\rho(t)<t$, left-scattered). If $\sigma(t)=t$, the point $t$ is said to be right-dense $(\rho(t)=t$, left-dense). Points that are simultaneously right-scattered and left-scattered are called isolated. The graininess function is defined by $\mu(t)=\sigma(t)-t$.

When $\mathbb{T}=\mathbb{R}$, then $\sigma(t)=t$, and $\mu(t)=0$; when $\mathbb{T}=\mathbb{Z}$, then $\sigma(t)=t+1$, and $\mu(t)=1$.
Definition 2 (see [14]). A function $f: \mathbb{T} \rightarrow \mathbb{R}$ has a limit $L$ at $t_{0} \in \mathbb{T}$ if and only if for every $\epsilon>0$ there exists $\delta>0$ such that if $t \in\left[t_{0}-\delta, t_{0}+\delta\right]$, then

$$
|f(t)-L|<\epsilon \text {. }
$$

If $t_{0}$ is an isolated point, then $L=f\left(t_{0}\right)$. If the limit exists, one writes

$$
\lim _{t \rightarrow t_{0}} f(t)=L \text {. }
$$

Definition 3. Consider $f: \mathbb{T} \rightarrow \mathbb{R}$, for each $t<\sup \mathbb{\mathbb { }}$, and define $f^{\Delta}(t)$ to be the number (provided it exists) with the property that, given any $\epsilon>0$, there is a neighborhood $U$ of $t$ such that

$$
\left|f(\sigma(t))-f(s)-f^{\Delta}(t)(\sigma(t)-s)\right| \leq \epsilon|\sigma(t)-s|,
$$

for all $s \in U . f^{\Delta}(t)$ is the delta-derivative of $f(t)$.

If $\mathbb{T}=\mathbb{R}$, then $f^{\Delta}(t)=f^{\prime}(t)$, the usual derivative, and if $\mathbb{T}=\mathbb{Z}$, then $f^{\Delta}(t)=f(t+1)-f(t)$, the forward difference operator.

Definition 4. If $F^{\Delta}(t)=f(t)$ and $t, t_{0} \in \mathbb{T}$, one defines the delta-integral by

$$
\int_{t_{0}}^{t} f(s) \Delta s=F(t)-F\left(t_{0}\right) .
$$

If $\mathbb{T}=\mathbb{R}$, then $\int_{t_{0}}^{t} f(s) \Delta s$ corresponds to the Cauchy integral $\int_{t_{0}}^{t} f(s) d s$ and if $\mathbb{T}=\mathbb{Z}$, then $\int_{t_{0}}^{t} f(s) \Delta s=\sum_{s=t_{0}}^{t-1} f(s)$.

Definition 5. A function $f: \mathbb{T} \rightarrow \mathbb{R}$ is right-dense (rd) continuous $\left(f \in C_{\mathrm{rd}}(\mathbb{T}, \mathbb{R})\right)$ if it is continuous at every rightdense point $t \in \mathbb{T}$ and $\lim _{s \rightarrow t^{-}} f(s)$ exists for every left-dense point $t \in \mathbb{T}$. Similarly, a function $f: \mathbb{T} \rightarrow \mathbb{R}$ is left-dense (ld) continuous $\left(f \in C_{\mathrm{ld}}(\mathbb{T}, \mathbb{R})\right)$ if it is continuous at every left-dense point $t \in \mathbb{T}$ and $\lim _{s \rightarrow t^{+}} f(s)$ exists for every rightdense point $t \in \mathbb{T}$.

Of course, every continuous function on $\mathbb{T}$ is also rdcontinuous and ld-continuous on $\mathbb{T}$. Furthermore, it is possible to prove (see [15]) that every rd-continuous function on $\mathbb{T}$ is delta-integrable on $\mathbb{T}$.

Let $\beta \in \mathbb{R}^{+}$; the exponential function $e_{\beta}\left(t, t_{0}\right), t \in \mathbb{T}$, is defined as the unique solution of the initial value problem (see, e.g., $[5,16]$ )

$$
\begin{aligned}
x^{\Delta} & =\beta x, \\
x\left(t_{0}\right) & =1 .
\end{aligned}
$$

The explicit form of $e_{\beta}\left(t, t_{0}\right)$ is given by

$$
\begin{aligned}
& e_{\beta}\left(t, t_{0}\right) \\
& \quad= \begin{cases}\exp \left(\beta\left(t-t_{0}\right)\right), & \text { if } \mu=0 \\
\exp \left(\int_{t_{0}}^{t} \frac{\ln (1+\mu(s) \beta)}{\mu(s)} \Delta s\right), & \text { if } \mu>0 .\end{cases}
\end{aligned}
$$


Observe that since $\beta>0$, we have $e_{\beta}\left(t, t_{0}\right)>0$ for all $t \epsilon$ $\left[t_{0},+\infty\right]_{\mathbb{T}}$. Furthermore, $e_{\beta}\left(t, t_{0}\right)$ is the solution of problem (10), so $e_{\beta}\left(t, t_{0}\right)^{\Delta}=\beta e_{\beta}\left(t, t_{0}\right)>0$; hence $e_{\beta}\left(t, t_{0}\right)$ is a strictly increasing function (see [3, Th. 1.76]) and

$$
\int_{t_{0}}^{t} e_{\beta}\left(s, t_{0}\right) \Delta s=\frac{1}{\beta} \int_{t_{0}}^{t} e_{\beta}^{\Delta}\left(s, t_{0}\right) \Delta s=\frac{e_{\beta}\left(t, t_{0}\right)-1}{\beta} .
$$

When $\mathbb{T}=\mathbb{R}$, then $e_{\beta}\left(t, t_{0}\right)=e^{\beta\left(t-t_{0}\right)}$, and if $\mathbb{T}=\mathbb{Z}$, then $e_{\beta}\left(t, t_{0}\right)=(1+\beta)^{t-t_{0}}$.

In the following it will be useful to define $C_{\beta}\left(\left[t_{0},+\infty\right]_{\mathbb{T}} ; \mathbb{R}\right)$ as the space of continuous functions $C\left(\left[t_{0},+\infty\right]_{\mathbb{T}} ; \mathbb{R}\right)$ such that

$$
\sup _{t \in\left[t_{0},+\infty\right]_{\mathbb{T}}} \frac{|x(t)|}{e_{\beta}\left(t, t_{0}\right)}<\infty .
$$

Let

$$
\|x\|_{\beta}=\sup _{t \in\left[t_{0},+\infty\right]_{\pi}} \frac{|x(t)|}{e_{\beta}\left(t, t_{0}\right)},
$$

the norm associated with $C_{\beta}\left(\left[t_{0},+\infty\right]_{\mathbb{T}} ; \mathbb{R}\right)$, and, for $\bar{t}<+\infty$, set

$$
\|x\|_{\beta}^{\bar{t}}=\sup _{t \in\left[t_{0}, \bar{t}\right]_{\mathbb{T}}} \frac{|x(t)|}{e_{\beta}\left(t, t_{0}\right)} .
$$

As already mentioned in the previous section, classical examples of time sets are $\mathbb{T}=\mathbb{R}$ and $\mathbb{T}=\mathbb{Z}$. Particularly useful from a theoretical point of view are the following time sets (see, e.g., [2]): $\mathbb{T}=q^{\mathbb{Z}}, \mathbb{T}=h \mathbb{Z}$, and $\mathbb{T}=\left\{t_{j}: j \in \mathbb{Z}\right\}$, which lead, respectively, to the $q$-difference equation

$$
x(t)=f(t)+(q-1) \sum_{j=\log _{q}\left(t_{0}\right)}^{\log _{q}(t)-1} q^{j} k\left(t, q^{j}\right) x\left(q^{j}\right),
$$

$$
q>1
$$

the Volterra discrete equation with constant stepsize

$$
x(t)=f(t)+h \sum_{j=t_{0} / h}^{t / h-1} k(t, j h) x(j h),
$$

and the discrete equation

$$
x\left(t_{n}\right)=f\left(t_{n}\right)+\sum_{j=0}^{n-1}\left(t_{j+1}-t_{j}\right) k\left(t_{n}, t_{j}\right) x\left(t_{j}\right) .
$$

In addition to the previous ones, examples are $\mathbb{T}=\left\{n^{2}: n \in\right.$ $\mathbb{N}\}, \mathbb{T}=[0,1] \cup \mathbb{N}, \mathbb{T}=\left\{\sum_{k=1}^{n}(1 / k): n \in \mathbb{N}\right\} \cup\{0\}$, and the biological relevant time scale

$$
\mathbb{T}=\bigcup_{k \in \mathbb{N}_{0}}[2 k, 2 k+1],
$$

where the life span of a certain species is supposed to be one unit of time and the time scale for simulating electric circuit is

$$
\mathbb{T}=\bigcup_{k \in \mathbb{N}_{0}}[k, k+1-\delta],
$$

where $\delta$ represents the time units for discharging the capacitor (see [3, Ex. 1.39, 1.40] for details).

\section{Stability and Convergence for Linear Equations}

In this section we investigate the boundedness of the solution to (1) when the forcing term $f(t)$ is bounded on $\left[t_{0},+\infty\right)_{\mathbb{T}}$. Since (1) is linear, it may be regarded as the error equation. Hence, our purpose here is to prove stability results for (1) under bounded perturbations, according to the following definition.

Definition 6. The zero solution $x(t)=0$ of the VIE on time scales (1) corresponding to $f(t)=0$ is called stable on $C\left(\left[t_{0},+\infty\right]_{\mathbb{1}} ; \mathbb{R}\right)$ if for each $\epsilon>0$ there exists a $\delta=\delta\left(\epsilon, t_{0}\right)>0$ such that $|f(t)| \leq \delta$ and $f(t) \in C\left(\left[t_{0},+\infty\right]_{\mathbb{T}} ; \mathbb{R}\right)$, implying that each solution $x(t)$ of (1) exists and satisfies $|x(t)| \leq \epsilon$, for all $t \geq t_{0}$.

From now on we assume that, in (1), the kernel $k$ : $\left[t_{0},+\infty\right)_{\mathbb{T}}^{2} \rightarrow \mathbb{R}$ is continuous with respect to the first variable and rd-continuous in the second variable. Furthermore, we assume that the forcing function $f$ is continuous on $\left[t_{0},+\infty\right)_{\mathbb{T}}$ (observe that, from Definition 2 , if $t$ is an isolated point, the definition of continuity is vacuously true).

In these hypotheses, if in addition $f \in C_{\beta}\left(\left[t_{0},+\infty\right)_{\mathbb{T}}, \mathbb{R}\right)$, Theorem 4.2 in [6] assures that the delta-integral equation (1) has a unique solution $x \in C_{\beta}\left(\left[t_{0},+\infty\right)_{\mathbb{T}}, R\right)$ and

$$
|x(t)| \leq X e_{\beta}\left(t, t_{0}\right),
$$

where $X$ is a positive constant. This bound, which is useful in applications (see [17, p. 37]), does not address the problem of the boundedness of the solution to $(1)$ on $\left[t_{0},+\infty\right)_{\mathbb{T}}$. In [6] it is shown that this is true under the additional hypothesis $\int_{t_{0}}^{t}|k(t, s)| \Delta s \leq m<1$, for all $t \in\left[t_{0},+\infty\right)_{\mathbb{T}}$. Here, we consider more general assumptions; that is,

(h1) $\sup _{t \in\left[t_{0},+\infty\right)_{\mathbb{T}}}|f(t)|<+\infty$;

(h2) $\sup _{t \in[s,+\infty)_{\mathbb{T}}}|k(t, s)|=\bar{K}<+\infty$, for each $s \geq t_{0}$;

(h3) $\exists \bar{t}>0$ such that $\sup _{t \in[\bar{t},+\infty)_{\mathbb{T}}} \int_{\bar{t}}^{t}|k(t, s)| \Delta s \leq \alpha<1$. and we prove that the solution to $(1)$ is bounded at $\left[t_{0},+\infty\right)_{\mathbb{T}}$.

Theorem 7. Assume that (h1)-(h3) hold. Then, there exists a constant $\Phi>0$ such that

$$
\sup _{t \in\left[t_{0},+\infty\right)_{\mathbb{T}}}|x(t)| \leq \frac{\Phi}{1-\alpha} \sup _{t \in\left[t_{0},+\infty\right)_{\mathbb{T}}}|f(t)| .
$$

Proof. Choose $\beta>0$ such that $\gamma=\bar{K} / \beta<1$, and consider the exponential function $e_{\beta}\left(t, t_{0}\right)$ defined in (11). Let $t<\bar{t}$; dividing each member of (1) by $e_{\beta}\left(t, t_{0}\right)$ one gets

$$
\begin{aligned}
& \frac{x(t)}{e_{\beta}\left(t, t_{0}\right)} \\
& =\frac{f(t)}{e_{\beta}\left(t, t_{0}\right)} \\
& \quad+\frac{1}{e_{\beta}\left(t, t_{0}\right)} \int_{t_{0}}^{t} k(t, s) e_{\beta}\left(s, t_{0}\right) \frac{x(s)}{e_{\beta}\left(s, t_{0}\right)} \Delta s .
\end{aligned}
$$


Thus, since (h1) and (h2) hold, by using the norm $\|\cdot\|_{\beta}^{\bar{t}}$ defined in (15) and the identity in (12), we get

$$
\begin{aligned}
\|x\|_{\beta}^{\bar{t}} & \leq\|f\|_{\beta}^{\bar{t}}+\|x\|_{\beta}^{\bar{t}} \bar{K} \frac{1}{e_{\beta}\left(t, t_{0}\right)} \frac{e_{\beta}\left(t, t_{0}\right)-1}{\beta} \\
& \leq\|f\|_{\beta}^{\bar{t}}+\|x\|_{\beta}^{\bar{t}} \frac{\bar{K}}{\beta} .
\end{aligned}
$$

Since $\gamma=\bar{K} / \beta<1$, then

$$
\|x\|_{\beta}^{\bar{t}} \leq \frac{\|f\|_{\beta}^{\bar{t}}}{1-\gamma} .
$$

When $t \geq \bar{t}(1)$ can be rewritten as

$$
x(t)=f(t)+\int_{t_{0}}^{\bar{t}} k(t, s) x(s) \Delta s+\int_{\bar{t}}^{t} k(t, s) x(s) \Delta s .
$$

Observing that $\int_{t_{0}}^{\bar{t}}|k(t, s)||x(s)| \Delta s \leq \bar{K} \int_{t_{0}}^{\bar{t}}\left(|x(s)| / e_{\beta}\left(s, t_{0}\right)\right)$ $e_{\beta}\left(s, t_{0}\right) \Delta s$, then result (25) can be used to obtain a bound $|x(s)| / e_{\beta}\left(s, t_{0}\right)$ and hence, since (h3) holds,

$$
\begin{aligned}
& \sup _{t \in\left[t_{0},+\infty\right)_{\top}}|x(t)| \\
& \leq \sup _{t \in\left[t_{0},+\infty\right)_{\top}}|f(t)|\left(1+\frac{\bar{K}}{1-\gamma} \frac{e_{\beta}\left(\bar{t}, t_{0}\right)-1}{\beta}\right) \\
& \quad+\alpha \sup _{t \in\left[t_{0},+\infty\right)_{\top}}|x(t)| .
\end{aligned}
$$

We know, from (h3), that $\alpha<1$; then

$$
\sup _{t \in\left[t_{0},+\infty\right)_{\mathbb{T}}}|x(t)| \leq \frac{\Phi}{1-\alpha} \sup _{t \in\left[t_{0},+\infty\right)_{\mathbb{T}}}|f(t)|,
$$

where $\Phi=1+(\bar{K} /(1-\gamma))\left(e_{\beta}\left(\bar{t}, t_{0}\right)-1 / \beta\right)$.

Remark 8. Boundedness results under hypothesis (h3) can be found, for example, in [11, Sec. 9, Th. 9.1] and in [12, 13] for nonconvolution VIEs $(\mathbb{T}=\mathbb{R})$ and Volterra summation equations $(\mathbb{T}=\mathbb{Z})$, respectively. The novelty here is that the different approach used in the proof of Theorem 7 allows the generalization to other kinds of time scales, as, for example, the ones in (19) and (20) motivated by the applications or $\mathbb{T}=h \mathbb{Z}$ motivated by numerical schemes. An analysis completely devoted to the stability of parameter-dependent Volterra summation equations has been carried out by the authors in [18].

Consider $\overline{\mathbb{T}}=\mathbb{\mathbb { V }} \cup\{\sup \mathbb{\mathbb { V }}\} \cup\{\inf \mathbb{\mathbb { T }}\}$. If $+\infty \in \overline{\mathbb{T}},+\infty$ is said to be left-dense (see $[15$, Sec. 4$]$ ). So we can consider $\lim _{t \rightarrow+\infty} y(t)$ for any function $y$ defined in $\left[t_{0},+\infty\right)_{\mathbb{T}}$. For the definition of lim sup on time scales we refer to [15, Sec. 4].

The following theorem is a generalization of Theorem 4.4 in [12] to Volterra equations on time scales.
Theorem 9. Assume that (h1)-(h3) hold. Furthermore, let

(h4) $\lim _{t \rightarrow+\infty} f(t)=f_{\infty}$,

(h5) $\lim _{t \rightarrow+\infty} k(t, s)=0, \forall s \in\left[t_{0},+\infty\right)_{\mathbb{T}}$,

(h6) $\lim _{\tau \rightarrow+\infty} \lim \sup _{t \rightarrow+\infty}\left|\int_{\tau}^{t} k(t, s) \Delta s-I_{k}\right|=0$, for some $I_{k}$.

Then $\lim _{t \rightarrow+\infty} x(t)=x_{\infty}$, where $x_{\infty}=f_{\infty} /\left(1-I_{k}\right)$.

Proof. First of all observe that, from (h3), $I_{k} \leq \alpha<1$. Manipulating (1) and subtracting $x_{\infty}=f_{\infty}+I_{k} x_{\infty}$, one gets

$$
\begin{aligned}
\left|x(t)-x_{\infty}\right| \leq & \left|f(t)-f_{\infty}\right|+\left|\int_{\tau}^{t} k(t, s) \Delta s-I_{k}\right|\left|x_{\infty}\right| \\
& +\int_{t_{0}}^{\tau}|k(t, s)||x(s)| \Delta s \\
& +\int_{\tau}^{t}|k(t, s)|\left|x(s)-x_{\infty}\right| \Delta s .
\end{aligned}
$$

Let us take the limit superior of each side of (29) as $t \rightarrow \infty$. Since $k$ is sufficiently smooth on $\left[t_{0},+\infty\right)_{\mathbb{T}} \times\left[t_{0},+\infty\right)_{\mathbb{T}}$ and hypotheses (h2) and (h5) hold, the limit can be passed under the integral sign in $\int_{t_{0}}^{\tau}|k(t, s)||x(s)| \Delta s$ to obtain zero (we refer to [19] for the Lebesgue bounded convergence theorem on time scales). Thus,

$$
\begin{aligned}
& \limsup _{t \rightarrow+\infty}\left|x(t)-x_{\infty}\right| \\
& \leq \lim _{t \rightarrow+\infty}\left|f(t)-f_{\infty}\right| \\
& \quad+\limsup _{t \rightarrow+\infty}\left|\int_{\tau}^{t} k(t, s) \Delta s-I_{k}\right|\left|x_{\infty}\right| \\
& \quad+\sup _{t \geq \tau}\left|x(t)-x_{\infty}\right| \sup _{t \geq \tau} \int_{\tau}^{t}|k(t, s)| \Delta s .
\end{aligned}
$$

By taking the lim sup for $\tau \rightarrow \infty$, in view of (h4), (h6), and (h3), we get the result.

\section{Extension to Nonlinear Equations}

For nonlinear equations of the form

$$
x(t)=f(t)+\int_{t_{0}}^{t} k(t, s) g(x(s)) \Delta s, \quad t \in\left[t_{0},+\infty\right]_{\mathbb{T}},
$$

we assume that $g$ is a differentiable map on $\mathbb{R}$ and $g(0)=0$. This condition is not restrictive; as a matter of fact, if this is not the case, we replace $g(x)$ with $g(x)-g(0)$ and $f(t)$ with $f(t)+\int_{t_{0}}^{t} k(t, s) g(0) \Delta s$.

Theorem 10. Assume that, for (31), $\left|g^{\prime}(x)\right| \leq G$, with $0 \leq G<$ $+\infty$, (h1) and (h2) hold, and

$$
\left(\mathrm{h} 3^{\prime}\right) \exists \bar{t}>0 \text { such that } \sup _{t \in[\bar{t},+\infty)_{\mathbb{T}}} G \int_{\bar{t}}^{t}|k(t, s)| \Delta s \leq \alpha<1 .
$$

Then the solution $x(t)$ to (31) is bounded. 
Proof. By Taylor's theorem we write $g(x)=g(0)+g^{\prime}(\xi) x$, $\xi \in(0, x)$. Since $g(0)=0$ and $\left|g^{\prime}(x)\right| \leq G$, for all $x$, from (31) one gets

$$
|x(t)| \leq|f(t)|+G \bar{K} \int_{t_{0}}^{t}|x(s)| \Delta s, \quad t \in\left[t_{0},+\infty\right]_{\mathbb{V}} .
$$

Then, for $t<\bar{t}$, by dividing each member of (32) by $e_{\beta}\left(t, t_{0}\right)$ it turns out that

$$
\|x\|_{\beta}^{\bar{t}} \leq\|f\|_{\beta}^{\bar{t}}+\|x\|_{\beta}^{\bar{t}} \frac{G \bar{K}}{\beta} .
$$

Choosing $\beta>0$ such that $\gamma_{G}=G \bar{K} / \beta<1$, then

$$
\|x\|_{\beta}^{\bar{t}} \leq \frac{\|f\|_{\beta}^{\bar{t}}}{1-\gamma_{G}}
$$

When $t \geq \bar{t}$, the following result is easily obtained:

$$
\sup _{t \in\left[t_{0},+\infty\right)_{\mathbb{T}}}|x(t)| \leq \frac{\Phi_{G}}{1-\alpha} \sup _{t \in\left[t_{0},+\infty\right)_{\mathbb{T}}}|f(t)|
$$

where $\Phi_{G}=1+\left(G \bar{K} /\left(1-\gamma_{G}\right)\right)\left(\left(e_{\beta}\left(\bar{t}, t_{0}\right)-1\right) / \beta\right)$.

\section{Examples}

For our examples we consider (1) with bounded $f(t)$ on $\left[t_{0},+\infty\right]_{\mathbb{T}}$ and

$$
k(t, s)=10 s e^{-s(t+1)},
$$

which does not satisfy the hypothesis $\int_{t_{0}}^{t}|k(t, s)| \Delta s<1$, considered in [6]. We study the stability of the solution for different choices of the time set $\mathbb{T}$.

$\mathbb{T}=\mathbb{R}$. Here $\int_{\bar{t}}^{t}|k(t, s)| \Delta s=\int_{\bar{t}}^{t}|k(t, s)| d s$. With $k$ defined as in (36) it is $|k(t, s)| \leq 10 s e^{-s}$ and $\int_{\bar{t}}^{t}|k(t, s)| d s \leq 10\left(e^{-\bar{t}}(1+\bar{t})\right)$, the last term in the inequality being a function in $\bar{t}$ which tends to zero as $\bar{t} \rightarrow \infty$. Hence $\int_{\bar{t}}^{t}|k(t, s)| d s<1$ for $\bar{t}>\overline{\bar{t}} \approx 3.9$.

$\mathbb{T}=\mathbb{Z}$. Here $\int_{\bar{t}}^{t}|k(t, s)| \Delta s=\sum_{s=\bar{t}}^{t-1}|k(t, s)|$. With $k$ defined as in (36) it is $|k(t, s)| \leq 10 s e^{-s}$ and, by simple manipulations, for $\bar{t}>1$, one gets $\sum_{s=\bar{t}}^{t-1}|k(t, s)| \leq 10\left(e^{2-\bar{t}} \bar{t}\right) /(e-1)^{2}$, the last term in the inequality being a function in $\bar{t}$ which tends to zero as $\bar{t} \rightarrow \infty$. Hence $\int_{\bar{t}}^{t}|k(t, s)| \Delta s<1$ for $\bar{t}>\overline{\bar{t}} \approx 4.8$.

$\mathbb{T}=h \mathbb{Z}$. Here $\int_{\bar{t}}^{t}|k(t, s)| \Delta s=h \sum_{j=\bar{t} / h}^{t / h-1}|k(t, j h)|$. By simple manipulations, for $\bar{t}>1$, one gets $h \sum_{s=\bar{t} / h}^{t / h-1}|k(t, s)| \leq$ $10 h\left(e^{2 h-\bar{t}} \bar{t}\right) /\left(e^{h}-1\right)^{2}$, the last term in the inequality being a function in $\bar{t}$ which tends to zero as $\bar{t} \rightarrow \infty$. Hence $\int_{\bar{t}}^{t}|k(t, s)| \Delta s<1$ for $\bar{t}>\overline{\bar{t}}$ depending on $h$. Of course, when $h=1$ we reduce to the case $\mathbb{T}=\mathbb{Z}$. For $h=0.1$ we get $\overline{\bar{t}} \approx 6.6$.

$\mathbb{T}=(19)$. Let $\bar{t}=2 \bar{k}$ and $t \in[2 N+2,2 N+3)$; then

$$
\begin{aligned}
\int_{\bar{t}}^{t}|k(t, s)| \Delta s= & \sum_{j=\bar{k}}^{N} \int_{2 j}^{2 j+1}|k(t, s)| d s \\
& +\int_{2 N+2}^{t}|k(t, s)| d s \\
& +\sum_{j=\bar{k}}^{N}|k(t, 2 j+1)| \\
\leq & \int_{\bar{t}}^{t}|k(t, s)| d s+\sum_{s=\bar{t}}^{t-1}|k(t, s)| .
\end{aligned}
$$

According to the discussion related to the cases $\mathbb{T}=\mathbb{R}$ and $\mathbb{T}=\mathbb{Z}$, when $k(t, s)$ is given by (36), it is possible to find $\overline{\bar{t}}$ such that for all $\bar{t}>\overline{\bar{t}}$ both $\int_{\bar{t}}^{t}|k(t, s)| d s$ and $\sum_{s=\bar{t}}^{t-1}|k(t, s)|$ are less than $1 / 2$. It turns out that this value for $\overline{\bar{t}}$ is $\approx 5.8$.

Then, the hypotheses of Theorem 7 are fulfilled in each of the time sets considered.

Observe that the kernel (36) satisfies also (h5) and (h6) for all the time sets considered above. So, for Theorem 9, $\lim _{t \rightarrow+\infty} x(t)=f_{\infty}$, if $\lim _{t \rightarrow+\infty} f(t)=f_{\infty}$.

\section{Concluding Remarks}

The research reported in this paper deals with the stability properties of Volterra equations on time scales. After examining the importance and the potential impact of this operator on the applications, we have surveyed the literature related to the calculus on time scales. As already mentioned in Section 1 and in Remark 8, in this paper, we extend some aspects of the stability theory, already developed in the continuous [11] and discrete [12] cases, to VIEs on time scales. Among the existing results for Volterra equations on time scales, the one in [6], concerning the existence and boundedness of the solutions to (1), has been our starting point for investigating their long time behavior. With respect to the results contained in [6], the boundedness of the solution is obtained here under more general sufficient conditions. This allows us to enlarge the class of problems under consideration, as shown in the example reported in Section 5 where kernel (36) is stable according to the current analysis, but does not satisfy the sufficient condition stated in [6].

The technique used in the proof of the main theorem put the basis for an analogous investigation about the numerical stability of Volterra integral equations.

\section{Conflict of Interests}

The authors declare that there is no conflict of interests regarding the publication of this paper. 


\section{Acknowledgments}

The authors thank the referee for his helpful suggestions. This work was supported by GNCS-INDAM.

\section{References}

[1] S. Hilger, "Analysis on measure chains. A unified approach to continuous and discrete calculus," Results in Mathematics, vol. 18, no. 1-2, pp. 18-56, 1990.

[2] R. P. Agarwal, M. Bohner, D. O'Regan, and A. Peterson, "Dynamic equations on time scales: a survey," Journal of Computational and Applied Mathematics, vol. 141, no. 1-2, pp. 1-26, 2002.

[3] M. Bohner and A. Peterson, Dynamic Equations on Time Scales. An Introduction with Applications, Birkhäauser, Boston, Mass, USA, 2001.

[4] M. Bohner and A. Peterson, Advances on Dynamic Equations on Time Scales, Birkhäuser, Boston, Mass, USA, 2003.

[5] C. C. Tisdell and A. Zaidi, "Basic qualitative and quantitative results for solutions to nonlinear, dynamic equations on time scales with an application to economic modelling," Nonlinear Analysis: Theory, Methods \& Applications, vol. 68, no. 11, pp. 3504-3524, 2008.

[6] T. Kulik and C. C. Tisdell, "Volterra integral equations on time scales: basic qualitative and quantitative results with applications to initial value problems on unbounded domains," International Journal of Difference Equations, vol. 3, no. 1, pp. 103-133, 2008.

[7] M. Adivar and Y. N. Raffoul, "Existence of resolvent for Volterra integral equations on time scales," Bulletin of the Australian Mathematical Society, vol. 82, no. 1, pp. 139-155, 2010.

[8] B. Karpuz, "Volterra theory on time scales," Results in Mathematics, vol. 65, no. 3-4, pp. 263-292, 2014.

[9] D. B. Pachpatte, "Properties of some dynamic integral equation on time scales," Annals of Functional Analysis, vol. 4, no. 2, pp. 12-26, 2013.

[10] I. L. D. dos Santos, "On Volterra integral equations on time scales," Mediterranean Journal of Mathematics, vol. 12, no. 2, pp. 471-480, 2015.

[11] G. Gripenberg, S.-O. Londen, and O. Staffans, Volterra Integral and Functional Equations, Encyclopedia of Mathematics and Its Applications, no. 34, Cambridge University Press, Cambridge, UK, 1990.

[12] I. Győri and D. W. Reynolds, "On admissibility of the resolvent of discrete Volterra equations," Journal of Difference Equations and Applications, vol. 16, no. 12, pp. 1393-1412, 2010.

[13] J. A. Applelby, I. Győri, and D. W. Reynolds, "On exact convergence rates for solutions of linear systems of Volterra difference equations," Journal of Difference Equations and Applications, vol. 12, no. 12, pp. 1257-1275, 2006.

[14] R. W. Oberste-Vorth, A. Mouzakitis, and B. A. Lawrence, Bridge to Abstract Mathematics, MAA, 2012.

[15] R. P. Agarwal and M. Bohner, "Basic calculus on time scales and some of its applications," Results in Mathematics, vol. 35, no. 1-2, pp. 3-22, 1999.

[16] M. Bohner and A. Peterson, "A survey of exponential function on times scales," Cubo: Matemática Educacional, vol. 3, no. 2, pp. 285-301, 2001.

[17] C. Corduneanu, Integral Equations and Stability of Feedback Systems, vol. 104 of Mathematics in Science and Engineering, Academic Press, New York, NY, USA, 1973.
[18] E. Messina, D. W. Reynolds, and A. Vecchio, "Parameterdependent Volterra summation equations," In preparation.

[19] G. A. Anastassiou, "Integral operator inequalities on time scales," International Journal of Difference Equations, vol. 7, no. 2, pp. 111-137, 2012. 


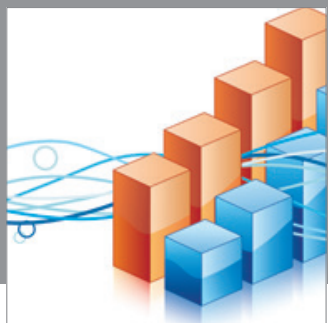

Advances in

Operations Research

mansans

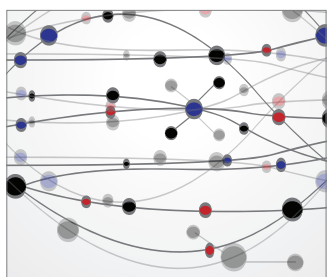

The Scientific World Journal
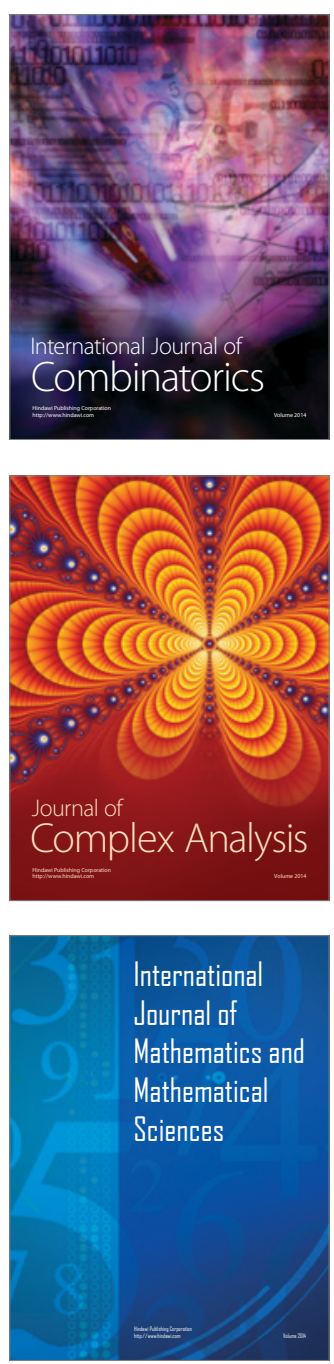
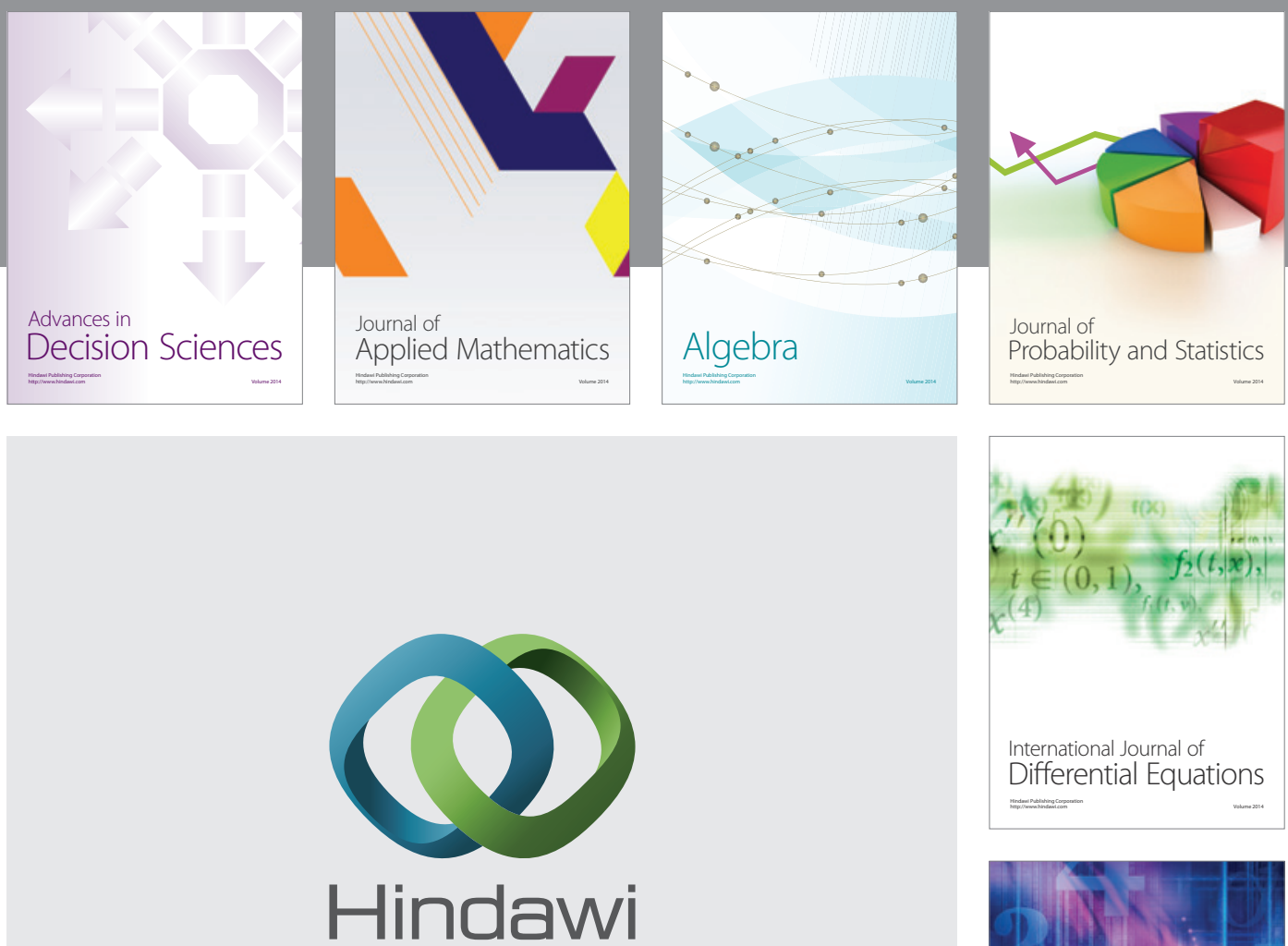

Submit your manuscripts at http://www.hindawi.com
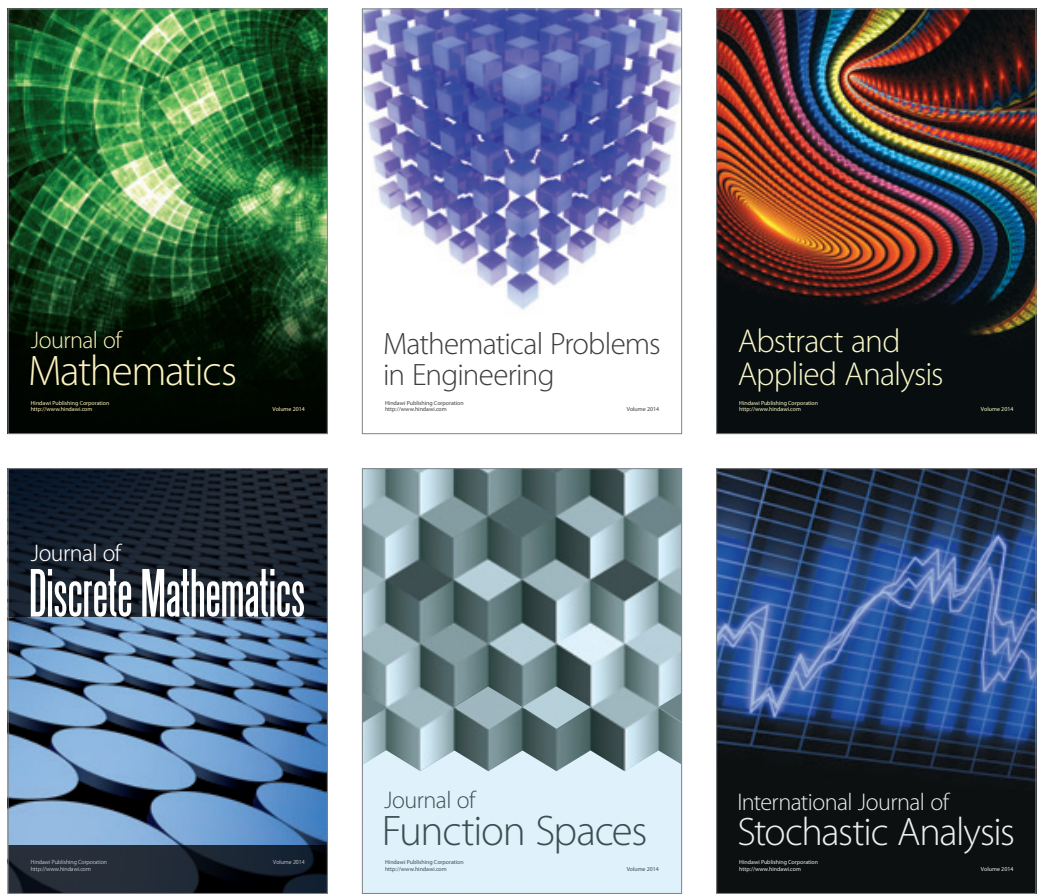

Journal of

Function Spaces

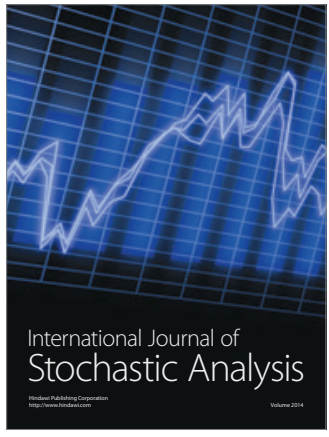

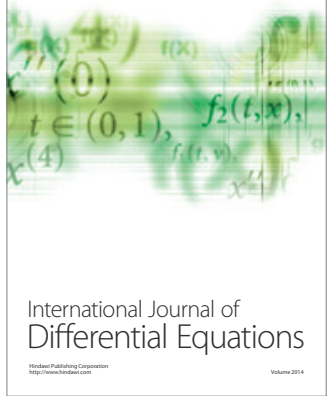
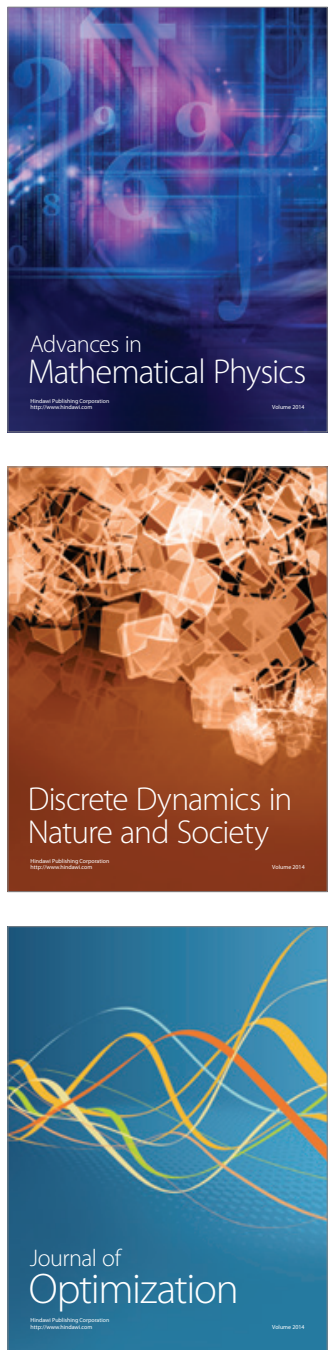